\title{
NATURAL DENSITY AND PROBABILITY, CONSTRUCTIVELY
}

\begin{abstract}
A b s t r a c t. We give a constructive account of the frequentist approach to probability, by means of natural density. Then we discuss some probabilistic variants of the Limited Principle of Omniscience.
\end{abstract}

\section{Introduction}

The notion of probability had different interpretations in its historical development. The naiff classical interpretation, according to which the probability of an event is given by the ratio of the number of favorable cases to the number of all possible cases, was extended at least in two directions: the first one is the frequentist approach, which consists in extending the classical interpretation from a finite number of possible cases to a sequence of outcomes ("success" or "failure") in a sequence of iterated trials; the second one is

Received 19 February 2019; resubmited 26 June 2019

Keywords and phrases: constructive probability, frequentist probability, natural density, limited principle of omniscience.

AMS Subject Classification: 03F65, 60A05. 
Kolmogorov's axiomatic approach, which consists in defining a probability measure as a $\sigma$-additive function defined on a $\sigma$-algebra of events.

Nowadays, in classical mathematics, Kolmogorov's axiomatic probability is the standard notion of probability. The frequentist approach is instead connected with the notion of natural density ([7]) which provides a notion of size for subsets of natural numbers. Classically, the density of $A \subseteq \mathbb{N}$ is defined as

$$
\delta(A)=\lim _{n \rightarrow \infty} \frac{|A \cap\{0, \ldots, n-1\}|}{n}
$$

provided that this limit exists. Since classically the subsets of $\mathbb{N}$ are in bijective correspondence with binary sequences (like "success"/"failure" sequences), this notion of density makes sense for such sequences, too.

Here we are interested in probability in an alternative mathematical framework, that is that of constructive mathematics in Bishop's sense ([1]). Bishop's mathematics can be roughly defined as mathematics based on intuitionistic logic. In his development of analysis, Bishop used only direct and constructive methods; for this reason its constructive analysis admits a computational interpretation. This means in particular that no principle like Law of Excluded Middle or Proof by Contraddiction can be used in constructive mathematics. Moreover in his Constructive Manifesto ([1]), E.Bishop was clear about constructivism: "The task of making analysis constructive is guided by three basic principles. First, to make every concept affirmative (...) Second, to avoid definitions that are not relevant (...) Third, to avoid pseudogenerality (...)".

In the context of constructive mathematics, the main reference for a treatment of probability is Chan's Notes on Constructive Probability ([3]). There, a constructive axiomatic definition of probability space (in the spirit of Kolmorogov's) is given. However this structure is not based on the notion of event, but on the notion of random number following the style of Daniell integral.

Here we will explore the frequentist interpretation of probability from a constructive point of view. We will exploit the sequential nature of events in the frequentist approach to define their probability in a very direct way; then we will prove some properties of such a notion of probability in Section 2.

Among the principles which are refused in Bishop's mathematics there is the so-called Limited Principle of Omniscience (LPO) for which every binary sequence is constantly 0 or has a term equal to 1 . Since our approach to natural density will naturally lead to the definition of some particular classes of binary sequences, it is a natural question to ask what is the relation between the several "probabilistic" variants of LPO which are obtained by 
restricting it to these classes and/or by weakening the statement $\forall n\left(x_{n}=0\right)$ by transforming it into $\mathbb{P}(x)=0$ and/or by strengthening the statement $\exists n\left(x_{n}=1\right)$ by transforming it into $\mathbb{P}(x)>0$. We will study these variants in Section 3 and we will prove there that each one of them is either true, false or equivalent to LPO itself, constructively.

Our treatment will be informal à la Bishop (see [1]), but it could be formalized in the extensional level of the Minimalist Foundation (see [6], [5]) with the addition of the axiom of unique choice and the axiom of countable choice.

\subsection{Some prerequisites}

Let us recall here the definition of Bishop real numbers from [1].

Definition 1.1. A Bishop real $x$ is a sequence $x(n) \in \mathbb{Q}\left[n \in \mathbb{N}^{+}\right]$of rational numbers such that

$$
|x(n)-x(m)| \leq \frac{1}{n}+\frac{1}{m}
$$

for every $n \in \mathbb{N}^{+}$and $m \in \mathbb{N}^{+}$.

Two Bishop reals $x$ and $y$ are equal if $|x(n)-y(n)| \leq \frac{2}{n}$ for every $n \in \mathbb{N}^{+}$. The set of Bishop reals is denoted with $\mathbb{R}$ and the equality between Bishop reals (which is an equivalence relation) is denoted with $=\mathbb{R}$.

We also recall how some basic operations between Bishop reals and their ordering are defined in [1]:

Definition 1.2. If $x$ and $y$ are Bishop reals, then

1. $x+y$ is the Bishop real defined by

$$
(x+y)(n):=x(2 n)+y(2 n)\left[n \in \mathbb{N}^{+}\right] ;
$$

2. $x-y$ is the Bishop real defined by

$$
(x-y)(n):=x(2 n)-y(2 n)\left[n \in \mathbb{N}^{+}\right] ;
$$

3. $x$ is non-positive if $x(n) \leq \frac{1}{n}$ for every $n \in \mathbb{N}^{+}$;

4. $x \leq_{\mathbb{R}} y$ if and only if $x-y$ is non-positive;

5. $x$ is positive if there exists $n \in \mathbb{N}^{+}$such that $x(n)>\frac{1}{n}$; 
6. $x<\mathbb{R} y$ if and only if $y-x$ is positive.

Moreover every rational number $q$ is identified, as a Bishop real, with the constant sequence $x$ such that $x(n)=q$ for every $n \in \mathbb{N}^{+}$.

\section{A constructive account of natural density}

In this section we will introduce a constructive notion of frequentist probability. We will draw a picture in which a structure of actual events (on which a notion of probability is defined) lies in between two nested Boolean algebras: that of potential events and that of regular events.

\subsection{Potential events}

A potential event e is a binary sequence

$$
\mathrm{e}(n) \in\{0,1\}\left[n \in \mathbb{N}^{+}\right]
$$

We interpret potential events as sequences of outcomes ("success" $=1$ or "failure" $=0$ ) in a sequence of iterated trials.

Potential events form a set with extensional equality, that is two potential events e and $\mathrm{e}^{\prime}$ are equal (we write $\mathrm{e}=_{\mathcal{P}} \mathrm{e}^{\prime}$ ) if $\mathrm{e}(n)=\mathrm{e}^{\prime}(n)$ for every $n \in \mathbb{N}^{+}$. This set, which we denote with $\mathcal{P}$, can be endowed with a structure of Boolean algebra as follows:

1. the bottom $\perp$ is $\lambda n .0$;

2. the top $\top$ is $\lambda n .1$;

3. the conjunction $\mathrm{e} \wedge \mathrm{e}^{\prime}$ of e and $\mathrm{e}^{\prime}$ in $\mathcal{P}$ is $\lambda n$. $\left(\mathrm{e}(n) \mathrm{e}^{\prime}(n)\right)$;

4. the disjunction of $\mathrm{e} \vee \mathrm{e}^{\prime}$ of $\mathrm{e}$ and $\mathrm{e}^{\prime}$ in $\mathcal{P}$ is $\lambda n .\left(\mathrm{e}(n)+\mathrm{e}^{\prime}(n)-\mathrm{e}(n) \mathrm{e}^{\prime}(n)\right)$;

5. the negation $\neg$ e of $\mathrm{e} \in \mathcal{P}$ is $\lambda n .(1-\mathrm{e}(n))$;

6. for every e, $\mathrm{e}^{\prime} \in \mathcal{P}, \mathrm{e} \leq \mathrm{e}^{\prime}$ if and only if $\mathrm{e}(n) \leq \mathrm{e}^{\prime}(n)$ for every $n \in \mathbb{N}^{+}$.

For every potential event e, one can define a sequence of rational numbers

$$
\Phi(\mathrm{e})(n) \in \mathbb{Q}\left[n \in \mathbb{N}^{+}\right]
$$

by taking $\Phi(\mathrm{e})(n)$ to be $\frac{\sum_{i=1}^{n} \mathrm{e}(i)}{n}$. This sequence is called the sequence of rates of success (or frequencies) of the potential event e. 


\section{$2.2 \quad$ Actual events}

Actual events are those potential events for which the sequence of rates of success can be shown constructively to converge to a Bishop real number.

Definition 2.1. An actual event is a pair $(e, \gamma)$ where e is a potential event and $\gamma$ is a strictly increasing sequence of natural numbers such that

$$
|\Phi(\mathrm{e})(\gamma(n)+i)-\Phi(\mathrm{e})(\gamma(n)+j)| \leq \frac{1}{n}
$$

for every $n \in \mathbb{N}^{+}, i \in \mathbb{N}$ and $j \in \mathbb{N}$.

We denote with $\widetilde{\mathcal{A}}$ the set of actual events and two actual events $(\mathrm{e}, \gamma)$ and $\left(\mathrm{e}^{\prime}, \gamma^{\prime}\right)$ are equal, $(\mathrm{e}, \gamma)=_{\widetilde{\mathcal{A}}}\left(\mathrm{e}^{\prime}, \gamma^{\prime}\right)$, if $\mathrm{e}={ }_{\mathcal{P}} \mathrm{e}^{\prime}$.

We now show that one can define a notion of probability for actual events. We first show that there exists a subsequence of the sequence of frequencies of an actual event which is a Bishop real.

Proposition 2.2. If $(\mathrm{e}, \gamma)$ is an actual event, then $\Phi(\mathrm{e}) \circ \gamma$ is a Bishop real.

Proof. Suppose $m \leq n$ are in $\mathbb{N}^{+}$. Then $\gamma(m) \leq \gamma(n)$ and hence

$$
|\Phi(\mathrm{e})(\gamma(n))-\Phi(\mathrm{e})(\gamma(m))| \leq \frac{1}{m}<\frac{1}{n}+\frac{1}{m} .
$$

This means that $\Phi(\mathrm{e}) \circ \gamma$ is a Bishop real.

Now we show that equal actual events give rise to equal Bishop reals.

Proposition 2.3. If $(\mathrm{e}, \gamma)$ and $\left(\mathrm{e}^{\prime}, \gamma^{\prime}\right)$ are equal actual events, then $\Phi(\mathrm{e}) \circ \gamma$ and $\Phi\left(\mathrm{e}^{\prime}\right) \circ \gamma^{\prime}$ are equal Bishop reals.

Proof. Suppose $(e, \gamma)$ and $\left(e^{\prime}, \gamma^{\prime}\right)$ are equal actual events. Since, for every positive natural number $n, \gamma(n) \leq \gamma^{\prime}(n)$ or $\gamma^{\prime}(n) \leq \gamma(n)$, and $\mathrm{e}=\mathcal{P} \mathrm{e}^{\prime}$, then for every $n \in \mathbb{N}^{+}$

$$
\left|(\Phi(\mathrm{e}) \circ \gamma)(n)-\left(\Phi\left(\mathrm{e}^{\prime}\right) \circ \gamma^{\prime}\right)(n)\right|=\left|\Phi(\mathrm{e})(\gamma(n))-\Phi(\mathrm{e})\left(\gamma^{\prime}(n)\right)\right| \leq \frac{1}{n}<\frac{2}{n} .
$$

This means that $\Phi(\mathrm{e}) \circ \gamma$ and $\Phi\left(\mathrm{e}^{\prime}\right) \circ \gamma^{\prime}$ are equal Bishop reals.

Thus we can give the following

Definition 2.4. The function $\mathbb{P}: \widetilde{\mathcal{A}} \rightarrow \mathbb{R}$ is defined as follows: if $(\mathrm{e}, \gamma)$ is an actual event, then $\mathbb{P}(\mathrm{e}, \gamma):=\Phi(\mathrm{e}) \circ \gamma$. The Bishop real number $\mathbb{P}(\mathrm{e}, \gamma)$ is called the probability of the actual event $(e, \gamma)$. 


\subsection{Properties of actual events}

We show here some basic properties of actual events and of the probability function $\mathbb{P}$ defined on them. However, before proceeding, we need the following

Lemma 2.5. If $(\mathrm{e}, \gamma)$ is an actual event and $\gamma^{\prime}$ is a strictly increasing sequence of positive natural numbers such that $\gamma(n) \leq \gamma^{\prime}(n)$ for every $n \in \mathbb{N}^{+}$, then $\left(\mathrm{e}, \gamma^{\prime}\right)$ is an actual event.

Proof. Let $n \in \mathbb{N}^{+}$and $i, j \in \mathbb{N}$. Then

$$
\begin{gathered}
\left|\Phi(\mathrm{e})\left(\gamma^{\prime}(n)+i\right)-\Phi(\mathrm{e})\left(\gamma^{\prime}(n)+j\right)\right| \\
=\left|\Phi(\mathrm{e})\left(\gamma(n)+\left(\gamma^{\prime}(n)-\gamma(n)+i\right)\right)-\Phi(\mathrm{e})\left(\gamma(n)+\left(\gamma^{\prime}(n)-\gamma(n)+j\right)\right)\right| \leq \frac{1}{n}
\end{gathered}
$$

since $\gamma^{\prime}(n)-\gamma(n) \geq 0$ and $(\mathrm{e}, \gamma)$ is an actual event.

First, we can prove that the probability of an actual event is always a value between 0 and 1 :

Proposition 2.6. If $(\mathrm{e}, \gamma)$ is an actual event, then $0 \leq \mathbb{R} \mathbb{P}(\mathrm{e}, \gamma) \leq \mathbb{R} 1$.

Proof. Let $(\mathrm{e}, \gamma)$ be an actual event. For every $m \in \mathbb{N}^{+}, 0 \leq \Phi(\mathrm{e})(m) \leq 1$. Thus, in particular, $-\Phi(\mathrm{e})(\gamma(2 n)) \leq \frac{1}{n}$ and $\Phi(\mathrm{e})(\gamma(2 n))-1 \leq \frac{1}{n}$ for every $n \in \mathbb{N}^{+}$. Hence $0 \leq_{\mathbb{R}} \mathbb{P}(\mathrm{e}, \gamma) \leq_{\mathbb{R}} 1$.

Moreover, $\mathbb{P}$ satisfies the property which is usually called strictness:

Proposition 2.7. $(\perp, \lambda n . n)$ is an actual event and $\mathbb{P}(\perp, \lambda n . n)=\mathbb{R}_{\mathbb{R}} 0$.

Proof. This follows from the fact that $\Phi(\perp)(n)=0$ for every $n \in \mathbb{N}^{+}$.

Then we show that actual events and $\mathbb{P}$ satisfy involution:

Proposition 2.8. If $(\mathrm{e}, \gamma)$ is an actual event, then $(\neg \mathrm{e}, \gamma)$ is an actual event and

$$
\mathbb{P}(\neg \mathrm{e}, \gamma)=\mathbb{R} 1-\mathbb{P}(\mathrm{e}, \gamma) .
$$

Proof. Let $(e, \gamma)$ be an actual event. Since $\Phi(\neg e)(n)=1-\Phi(e)(n)$ for every $n \in \mathbb{N}^{+}$, it follows that for every $n \in \mathbb{N}^{+}$and $m, m^{\prime} \in \mathbb{N}$

$$
\begin{aligned}
& \left|\Phi(\neg \mathrm{e})(\gamma(n)+m)-\Phi(\neg \mathrm{e})\left(\gamma(n)+m^{\prime}\right)\right| \\
= & \left|\Phi(\mathrm{e})\left(\gamma(n)+m^{\prime}\right)-\Phi(\mathrm{e})(\gamma(n)+m)\right| \leq \frac{1}{n} .
\end{aligned}
$$


Hence $(\neg e, \gamma)$ is an actual event. Moreover for every $n \in \mathbb{N}^{+}$

$$
\begin{aligned}
\mid \mathbb{P}(\neg \mathrm{e}, \gamma)(n)- & (1-\mathbb{P}(\mathrm{e}, \gamma))(n)|=| 1-\mathbb{P}(\mathrm{e}, \gamma)(n)-(1-\mathbb{P}(\mathrm{e}, \gamma)(2 n)) \mid \\
& =|\mathbb{P}(\mathrm{e}, \gamma)(2 n)-\mathbb{P}(\mathrm{e}, \gamma)(n)| \leq \frac{1}{n}+\frac{1}{2 n}<\frac{2}{n}
\end{aligned}
$$

so $\mathbb{P}(\neg \mathrm{e}, \gamma)=\mathbb{R} 1-\mathbb{P}(\mathrm{e}, \gamma)$.

Moreover actual events are closed under disjunction of incompatible events:

Proposition 2.9. If $(\mathrm{e}, \gamma)$ and $\left(\mathrm{e}^{\prime}, \gamma^{\prime}\right)$ are actual events with $\mathrm{e} \wedge \mathrm{e}^{\prime}={ }_{\mathcal{P}} \perp$ and $\eta:=\lambda n \cdot\left(\gamma(2 n)+\gamma^{\prime}(2 n)\right)$, then the pair $\left(\mathrm{e} \vee \mathrm{e}^{\prime}, \eta\right)$ is an actual event.

Proof. Since e $\wedge \mathrm{e}^{\prime}=_{\mathcal{P}} \perp$, we have that $\mathrm{e} \vee \mathrm{e}^{\prime}=_{\mathcal{P}} \mathrm{e}+\mathrm{e}^{\prime}$. Moreover $\eta$ is stricly increasing, as $\gamma$ and $\gamma^{\prime}$ are so, and, for every $n \in \mathbb{N}^{+}$, we have that

$$
\Phi\left(\mathrm{e}+\mathrm{e}^{\prime}\right)(n)=\Phi(\mathrm{e})(n)+\Phi\left(\mathrm{e}^{\prime}\right)(n) .
$$

In particular, for every $n \in \mathbb{N}^{+}, i, j \in \mathbb{N}$, using subadditivity of the absolute value, we have that

$$
\begin{aligned}
& \left|\Phi\left(\mathrm{e}+\mathrm{e}^{\prime}\right)(\eta(n)+i)-\Phi\left(\mathrm{e}+\mathrm{e}^{\prime}\right)(\eta(n)+j)\right| \\
& \leq \quad\left|\Phi(\mathrm{e})\left(\gamma(2 n)+\gamma^{\prime}(2 n)+i\right)-\Phi(\mathrm{e})\left(\gamma(2 n)+\gamma^{\prime}(2 n)+j\right)\right| \\
& \quad+\left|\Phi\left(\mathrm{e}^{\prime}\right)\left(\gamma^{\prime}(2 n)+\gamma(2 n)+i\right)-\Phi\left(\mathrm{e}^{\prime}\right)\left(\gamma^{\prime}(2 n)+\gamma(2 n)+j\right)\right| \\
& \leq \quad \frac{1}{2 n}+\frac{1}{2 n}=\frac{1}{n} .
\end{aligned}
$$

Hence $\left(e \vee e^{\prime}, \eta\right)$ is an actual event.

We now show that the set of actual events with null probability is downward closed:

Proposition 2.10. Let $(\mathrm{e}, \gamma)$ be an actual event with $\mathbb{P}(\mathrm{e}, \gamma)=\mathbb{R}_{\mathbb{R}} 0$ and let $\mathrm{e}^{\prime}$ be a potential event such that $\mathrm{e}^{\prime} \leq \mathrm{e}$, then $\left(\mathrm{e}^{\prime}, \lambda n . \gamma(6 n)\right)$ is an actual event.

Proof. First notice that if $\mathrm{e}^{\prime} \leq \mathrm{e}$, then $\Phi\left(\mathrm{e}^{\prime}\right)(n) \leq \Phi(\mathrm{e})(n)$ for every $n \in \mathbb{N}^{+}$. 
Thus, for every $n \in \mathbb{N}^{+}$and $i, j \in \mathbb{N}$, using the subadditivity of the absolute value function and an add-subtract trick, we have

$$
\begin{aligned}
&\left|\Phi\left(\mathrm{e}^{\prime}\right)(\gamma(6 n)+i)-\Phi\left(\mathrm{e}^{\prime}\right)(\gamma(6 n)+j)\right| \\
& \leq\left|\Phi\left(\mathrm{e}^{\prime}\right)(\gamma(6 n)+i)\right|+\left|\Phi\left(\mathrm{e}^{\prime}\right)(\gamma(6 n)+j)\right| \\
& \leq|\Phi(\mathrm{e})(\gamma(6 n)+i)|+|\Phi(\mathrm{e})(\gamma(6 n)+j)| \\
& \leq|\Phi(\mathrm{e})(\gamma(6 n)+i)-\Phi(\mathrm{e})(\gamma(6 n))|+|\Phi(\mathrm{e})(\gamma(6 n)+j)-\Phi(\mathrm{e})(\gamma(6 n))| \\
&+2|\Phi(\mathrm{e})(\gamma(6 n))| \\
& \leq \frac{1}{6 n}+\frac{1}{6 n}+2 \frac{2}{6 n}=\frac{1}{n} .
\end{aligned}
$$

The last inequality follows from the fact that $(e, \gamma)$ is an actual event and $\mathbb{P}(\mathrm{e}, \gamma)=_{\mathbb{R}} 0$. Hence $\left(\mathrm{e}^{\prime}, \lambda n . \gamma(6 n)\right)$ is an actual event.

Moreover $\mathbb{P}$ satisfies monotonicity:

Proposition 2.11. If $(\mathrm{e}, \gamma)$ and $\left(\mathrm{e}^{\prime}, \gamma^{\prime}\right)$ are actual events and $\mathrm{e} \leq \mathrm{e}^{\prime}$, then

$$
\mathbb{P}(\mathrm{e}, \gamma) \leq_{\mathbb{R}} \mathbb{P}\left(\mathrm{e}^{\prime}, \gamma^{\prime}\right) .
$$

Proof. First of all if we take $\eta$ to be the sequence $\lambda n .\left(\gamma(n)+\gamma^{\prime}(n)\right)$, then, by Lemma $2.5,(\mathrm{e}, \eta)$ and $\left(\mathrm{e}^{\prime}, \eta\right)$ are actual events (equal by definition to $(\mathrm{e}, \gamma)$ and $\left(\mathrm{e}^{\prime}, \gamma^{\prime}\right)$, respectively). In order to show that $\mathbb{P}(\mathrm{e}, \eta) \leq_{\mathbb{R}} \mathbb{P}\left(\mathrm{e}^{\prime}, \eta\right)$, we must prove that $\left(\mathbb{P}(\mathrm{e}, \eta)-\mathbb{P}\left(\mathrm{e}^{\prime}, \eta\right)\right)(n) \leq \frac{1}{n}$ for every $n \in \mathbb{N}^{+}$. But $\left(\mathbb{P}(\mathrm{e}, \eta)-\mathbb{P}\left(\mathrm{e}^{\prime}, \eta\right)\right)(n)$ is equal to

$$
\mathbb{P}(\mathrm{e}, \eta)(2 n)-\mathbb{P}\left(\mathrm{e}^{\prime}, \eta\right)(2 n)=\Phi(\mathrm{e})(\eta(2 n))-\Phi\left(\mathrm{e}^{\prime}\right)(\eta(2 n)) .
$$

Hence we must prove that $\Phi(\mathrm{e})(\eta(2 n)) \leq \Phi\left(\mathrm{e}^{\prime}\right)(\eta(2 n))+\frac{1}{n}$ for every $n \in \mathbb{N}^{+}$. But this is true, because from $\mathrm{e} \leq \mathrm{e}^{\prime}$ we deduce that $\Phi(\mathrm{e})(n) \leq \Phi\left(\mathrm{e}^{\prime}\right)(n)$ for every positive natural number $n$.

Finally we prove that $\mathbb{P}$ satisfies a form of modularity:

Proposition 2.12. If $\left(\mathrm{e}, \gamma_{1}\right),\left(\mathrm{e}^{\prime}, \gamma_{2}\right),\left(\mathrm{e} \wedge \mathrm{e}^{\prime}, \gamma_{3}\right),\left(\mathrm{e} \vee \mathrm{e}^{\prime}, \gamma_{4}\right)$ are actual events, then

$$
\mathbb{P}\left(\mathrm{e} \vee \mathrm{e}^{\prime}, \gamma_{4}\right)+\mathbb{P}\left(\mathrm{e} \wedge \mathrm{e}^{\prime}, \gamma_{3}\right)={ }_{\mathbb{R}} \mathbb{P}\left(\mathrm{e}, \gamma_{1}\right)+\mathbb{P}\left(\mathrm{e}^{\prime}, \gamma_{2}\right) .
$$

Proof. Let $\varepsilon:=\lambda n \cdot\left(\gamma_{1}(n)+\gamma_{2}(n)+\gamma_{3}(n)+\gamma_{4}(n)\right)$. Since $\varepsilon(n) \geq \gamma_{i}(n)$ for every $n \in \mathbb{N}^{+}$for $i=1,2,3,4$, we can use Lemma 2.5 and obtain that:

1. $\mathbb{P}\left(\mathrm{e} \vee \mathrm{e}^{\prime}, \gamma_{4}\right)+\mathbb{P}\left(\mathrm{e} \wedge \mathrm{e}^{\prime}, \gamma_{3}\right)=\mathbb{R} \mathbb{P}\left(\mathrm{e} \vee \mathrm{e}^{\prime}, \varepsilon\right)+\mathbb{P}\left(\mathrm{e} \wedge \mathrm{e}^{\prime}, \varepsilon\right)$ 
2. $\mathbb{P}\left(\mathrm{e}, \gamma_{1}\right)+\mathbb{P}\left(\mathrm{e}^{\prime}, \gamma_{2}\right)=\mathbb{R} \mathbb{P}(\mathrm{e}, \varepsilon)+\mathbb{P}\left(\mathrm{e}^{\prime}, \varepsilon\right)$

In order to conclude, it is sufficient to prove that

$$
\mathbb{P}(\mathrm{e}, \varepsilon)+\mathbb{P}\left(\mathrm{e}^{\prime}, \varepsilon\right)=_{\mathbb{R}} \mathbb{P}\left(\mathrm{e} \vee \mathrm{e}^{\prime}, \varepsilon\right)+\mathbb{P}\left(\mathrm{e} \wedge \mathrm{e}^{\prime}, \varepsilon\right) .
$$

This follows from the fact that $\mathrm{e}(n)+\mathrm{e}^{\prime}(n)=\left(\mathrm{e} \vee \mathrm{e}^{\prime}\right)(n)+\left(\mathrm{e} \wedge \mathrm{e}^{\prime}\right)(n)$ for every $n \in \mathbb{N}^{+}$from which it follows that $\Phi(\mathrm{e})(n)+\Phi\left(\mathrm{e}^{\prime}\right)(n)=\Phi\left(\mathrm{e} \wedge \mathrm{e}^{\prime}\right)(n)+$ $\Phi\left(\mathrm{e} \vee \mathrm{e}^{\prime}\right)(n)$ for every $n \in \mathbb{N}^{+}$.

\subsection{Regular events}

Among potential events, there are some events which can be considered sort of "deterministic", since their sequences of outcomes of trials have a periodic behaviour, up to a possible finite number of accidental errors in the recording of the results. These events are here called regular.

Definition 2.13. Let $\underline{\pi}$ be a finite non-empty list of elements of $\{0,1\}$ with lenght $\ell(\underline{\pi})$. We define the potential event $\underline{\pi}^{\infty}$ as the periodic sequence defined by

$$
\underline{\pi}^{\infty}(n):=\pi_{\bmod (n-1, \ell(\underline{\pi}))+1}\left[n \in \mathbb{N}^{+}\right]
$$

where we denote with $\pi_{i}$ the $i$-th component of the list $\underline{\pi}$.

Definition 2.14. Let $\underline{\alpha}$ and $\underline{\pi}$ be two finite lists of elements of $\{0,1\}$ with $\underline{\pi}$ non-empty, and let $\ell(\underline{\alpha})$ and $\ell(\underline{\pi})$ be their lenght, respectively. The potential event $\underline{\alpha} \cdot \underline{\pi}^{\infty}$ is a sequence having $\underline{\alpha}$ as prefix and then having period $\underline{\pi}$, that is the ultimately periodic sequence defined as follows:

$$
\left\{\begin{array}{l}
\left(\underline{\alpha} \cdot \underline{\pi}^{\infty}\right)(n):=\alpha_{n}\left[n \in \mathbb{N}^{+}, n \leq \ell(\underline{\alpha})\right] \\
\left(\underline{\alpha} \cdot \underline{\pi}^{\infty}\right)(n):=\underline{\pi}^{\infty}(n-\ell(\underline{\alpha}))\left[n \in \mathbb{N}^{+}, n>\ell(\underline{\alpha})\right]
\end{array}\right.
$$

where we denote with $\alpha_{i}$ the $i$-th component of the list $\underline{\alpha}$. The potential events of this form are called regular.

Notice that every periodic binary sequence $\underline{\pi}^{\infty}$ is regular, since $\underline{\pi}^{\infty}=[] \cdot \underline{\pi}^{\infty}$.

The goal of the following propositions is to show that regular events are actual. First we show that regular events without errors (that is periodic sequences) are actual events and their probability is equal to the frequency of their period. 
Proposition 2.15. For every finite non-empty list $\underline{\pi}=\left[\pi_{1}, \ldots, \pi_{m}\right]$, $\left(\underline{\pi}^{\infty}, \lambda n .4 n m\right)$ is an actual event and

$$
\mathbb{P}\left(\underline{\pi}^{\infty}, \lambda n .4 n m\right)={ }_{\mathbb{R}} \frac{\sum_{k=1}^{m} \pi_{k}}{m} .
$$

Proof. For every $n \in \mathbb{N}^{+}$and every $i, j \in \mathbb{N}$, using the subadditivity of the absolute value function, we obtain

$$
\begin{aligned}
&\left|\Phi\left(\underline{\pi}^{\infty}\right)(4 n m+i)-\Phi\left(\underline{\pi}^{\infty}\right)(4 n m+j)\right| \\
&=\mid \frac{\sum_{k=1}^{m} \pi_{k}}{m}\left(\frac{\left(4 n+\left\lfloor\frac{i}{m}\right\rfloor\right) m}{4 n m+i}\right)+\frac{\sum_{k=1}^{\bmod (i, m)} \pi_{k}}{4 n m+i} \\
&-\frac{\sum_{k=1}^{m} \pi_{k}}{m}\left(\frac{\left(4 n+\left\lfloor\frac{j}{m}\right\rfloor\right) m}{4 n m+j}\right)-\frac{\sum_{k=1}^{\bmod (j, m)} \pi_{k}}{4 n m+j} \mid \\
& \leq \frac{\sum_{k=1}^{m} \pi_{k}}{m}\left|\frac{\left(4 n+\left\lfloor\frac{i}{m}\right\rfloor\right) m}{4 n m+i}-\frac{\left(4 n+\left\lfloor\frac{j}{m}\right\rfloor\right) m}{4 n m+j}\right| \\
&+\left|\frac{\sum_{k=1}^{\bmod (i, m)}}{4 n m+i}+\frac{\sum_{k=1}^{\bmod (j, m)} \pi_{k}}{4 n m+j}\right| \\
& \leq\left|\frac{\left(4 n+\left\lfloor\frac{i}{m}\right\rfloor\right) m}{4 n m+i}-\frac{\left(4 n+\left\lfloor\frac{j}{m}\right\rfloor\right) m}{4 n m+j}\right|+\left|\frac{2 \sum_{k=1}^{m} \pi_{k}}{4 n m}\right| \\
& \leq 1-\frac{4 n}{4 n+1}+\frac{1}{2 n}=\frac{1}{4 n+1}+\frac{1}{2 n}<\frac{1}{n}
\end{aligned}
$$

since for every $n, m \in \mathbb{N}^{+}$and for every $i \in \mathbb{N}$

$$
\frac{4 n}{4 n+1} \leq \frac{\left(4 n+\left\lfloor\frac{i}{m}\right\rfloor\right) m}{4 n m+i} \leq 1 .
$$

Hence $\left(\underline{\pi}^{\infty}, \lambda n .4 n m\right)$ is an actual event. Moreover for every $i \in \mathbb{N}^{+}$

$$
\mathbb{P}\left(\underline{\pi}^{\infty}, \lambda n .4 n m\right)(i)=\frac{\sum_{k=1}^{m} \pi_{k}}{m} .
$$

Hence $\mathbb{P}\left(\underline{\pi}^{\infty}, \lambda n .4 n m\right)=\mathbb{R} \frac{\sum_{k=1}^{m} \pi_{k}}{m}$.

The next step consists in proving that regular events definitely equal to 0 are actual events and their probability is 0 .

Proposition 2.16. If $\underline{\alpha}$ is a finite list of $0 s$ and $1 s$ with length $m>0$, then $\left(\underline{\alpha} .[0]^{\infty}, \lambda n .2 n m\right)$ is an actual event and $\mathbb{P}\left(\underline{\alpha} .[0]^{\infty}, \lambda n .2 n m\right)=\mathbb{R}^{0}$. 
Proof. Suppose $n \in \mathbb{N}^{+}$and $i, j \in \mathbb{N}$ with $i \geq j$. Then, using the subadditivity of the absolute value function,

$$
\begin{aligned}
& \left|\Phi\left(\underline{\alpha} \cdot[0]^{\infty}\right)(2 n m+i)-\Phi\left(\underline{\alpha} \cdot[0]^{\infty}\right)(2 n m+j)\right| \\
& \quad \leq\left|\Phi\left(\underline{\alpha} \cdot[0]^{\infty}\right)(2 n m+i)\right|+\left|\Phi\left(\underline{\alpha} \cdot[0]^{\infty}\right)(2 n m+j)\right| \\
& \quad \leq \frac{\alpha_{1}+\ldots+\alpha_{m}}{2 n m+i}+\frac{\alpha_{1}+\ldots+\alpha_{m}}{2 n m+j} \leq \frac{2 m}{2 n m}=\frac{1}{n} .
\end{aligned}
$$

Hence $\left(\underline{\alpha} .[0]^{\infty}, \lambda n .2 n m\right)$ is an actual event. Moreover for every $n \in \mathbb{N}^{+}$

$$
\mathbb{P}\left(\underline{\alpha} \cdot[0]^{\infty}, \lambda n .2 n m\right)(n)=\frac{\alpha_{1}+\ldots+\alpha_{m}}{2 n m} \leq \frac{m}{2 n m}=\frac{1}{2 n}<\frac{2}{n}
$$

Thus $\mathbb{P}\left(\underline{\alpha} \cdot[0]^{\infty}, \lambda n .2 n m\right)=_{\mathbb{R}} 0$.

Finally we prove that actual events are closed under shift to the right of terms and that probability is preserved by these shifts.

Definition 2.17. If e is a potential event, then $\mathrm{e}^{+}$is the potential event defined by

$$
\left\{\begin{array}{l}
\mathrm{e}^{+}(1):=0, \\
\mathrm{e}^{+}(n+1):=\mathrm{e}(n)\left[n \in \mathbb{N}^{+}\right] .
\end{array}\right.
$$

Proposition 2.18. If $(\mathrm{e}, \gamma)$ is an actual event, then $\left(\mathrm{e}^{+}, \lambda n \cdot(\gamma(3 n)+1)\right)$ is an actual event and $\mathbb{P}\left(\mathrm{e}^{+}, \lambda n \cdot(\gamma(3 n)+1)\right)=\mathbb{R} \mathbb{P}(\mathrm{e}, \gamma)$.

Proof. Using an add-subtract trick and the subadditivity of the absolute value function, we obtain that for $n \in \mathbb{N}^{+}$and $i, j \in \mathbb{N}$, the value

$$
\left|\Phi\left(\mathrm{e}^{+}\right)(\gamma(3 n)+1+i)-\Phi\left(\mathrm{e}^{+}\right)(\gamma(3 n)+1+j)\right|
$$

is less or equal than the sum of

1. $\left|\Phi\left(\mathrm{e}^{+}\right)(\gamma(3 n)+1+i)-\Phi(\mathrm{e})(\gamma(3 n)+i)\right|$,

2. $|\Phi(\mathrm{e})(\gamma(3 n)+i)-\Phi(\mathrm{e})(\gamma(3 n)+j)|$ and

3. $\left|\Phi(\mathrm{e})(\gamma(3 n)+j)-\Phi\left(\mathrm{e}^{+}\right)(\gamma(3 n)+1+j)\right|$.

Since for every $m \in \mathbb{N}$

$$
\Phi\left(\mathrm{e}^{+}\right)(m+1)=\frac{\sum_{k=1}^{m+1} \mathrm{e}^{+}(k)}{m+1}=\frac{\sum_{k=1}^{m} \mathrm{e}(k)}{m+1}=\frac{m \cdot \Phi(\mathrm{e})(m)}{m+1}
$$


the sum above is equal to

$$
\frac{\Phi(\mathrm{e})(\gamma(3 n)+i)}{\gamma(3 n)+i+1}+|\Phi(\mathrm{e})(\gamma(3 n)+i)-\Phi(\mathrm{e})(\gamma(3 n)+j)|+\frac{\Phi(\mathrm{e})(\gamma(3 n)+j)}{\gamma(3 n)+j+1}
$$

which is less or equal to

$$
\frac{1}{\gamma(3 n)+i+1}+\frac{1}{3 n}+\frac{1}{\gamma(3 n)+j+1} \leq 3 \frac{1}{3 n}=\frac{1}{n}
$$

since $\Phi(\mathrm{e})(m) \leq 1$ for every $m \in \mathbb{N}^{+}, \gamma(3 n) \geq 3 n$ (because $\gamma$ is strictly increasing $)$ and $(\mathrm{e}, \gamma)$ is an actual event. Hence $\left(\mathrm{e}^{+}, \lambda n \cdot(\gamma(3 n)+1)\right)$ is an actual event. Moreover, using the subadditivity of the absolute value function

$$
\begin{aligned}
\mid \Phi & \left(\mathrm{e}^{+}\right)(\gamma(3 n)+1)-\Phi(\mathrm{e})(\gamma(n)) \mid \\
& =\left|\Phi(\mathrm{e})(\gamma(3 n)) \frac{\gamma(3 n)}{\gamma(3 n)+1}-\Phi(\mathrm{e})(\gamma(n))\right| \\
& =\left|\Phi(\mathrm{e})(\gamma(3 n))-\Phi(\mathrm{e})(\gamma(n))-\frac{\Phi(\mathrm{e})(\gamma(3 n))}{\gamma(3 n)+1}\right| \\
& \leq|\Phi(\mathrm{e})(\gamma(3 n))-\Phi(\mathrm{e})(\gamma(n))|+\left|\Phi(\mathrm{e})(\gamma(3 n)) \frac{1}{\gamma(3 n)+1}\right| \\
& \leq \frac{1}{n}+\frac{1}{3 n+1}<\frac{2}{n} .
\end{aligned}
$$

Hence $\mathbb{P}\left(\mathrm{e}^{+}, \lambda n \cdot(\gamma(3 n)+1)\right)=\mathbb{R} \mathbb{P}(\mathrm{e}, \gamma)$.

Putting these results together we obtain the following:

Theorem 2.19. For every regular event $\underline{\alpha} \cdot \underline{\pi}^{\infty}$, there exists a strictly increasing sequence of natural numbers $\gamma$ such that $\left(\underline{\alpha} \cdot \underline{\pi}^{\infty}, \gamma\right)$ is an actual event and

$$
\mathbb{P}\left(\underline{\alpha} \cdot \underline{\pi}^{\infty}, \gamma\right)=\frac{\sum_{k=1}^{\ell(\underline{\pi})} \pi_{k}}{\ell(\underline{\pi})} .
$$

Proof. If $\underline{\alpha}$ is empty, then the result follows from Proposition 2.15. So we can assume $\underline{\alpha}$ to be non-empty.

First of all, notice that $\underline{\alpha} \cdot \underline{\pi}^{\infty}=\mathcal{P} \underline{\alpha} \cdot[0]^{\infty} \vee \underline{0}^{\ell(\alpha)} \cdot \underline{\pi}^{\infty}$ where we denoted with $\underline{0}^{\ell(\underline{\alpha})}$ a list of 0 s of leght $\ell(\underline{\alpha})$. Moreover $\underline{0}^{\ell(\underline{\alpha})} \cdot \underline{\pi}^{\infty}$ is the result of applying $\ell(\underline{\alpha})$ times $(-)^{+}$to $\underline{\pi}^{\infty}$. Thus, as a consequence of Propositions 2.15 and 2.18, there exists $\gamma_{2}$ such that $\left(\underline{0}^{\ell}(\underline{\alpha}) \cdot \underline{\pi}^{\infty}, \gamma_{2}\right)$ is an actual event. By Proposition 2.16, we also know that there exists $\gamma_{1}$ such that $\left(\underline{\alpha} .[0]^{\infty}, \gamma_{1}\right)$ is an actual event. 
Since $\underline{\alpha} \cdot[0]^{\infty} \wedge \underline{Q}^{\ell} \underline{(\alpha)} \cdot \underline{\pi}=\mathcal{P} \perp$, using Proposition 2.9, we obtain that there exists $\gamma$ such that $\left(\underline{\alpha} \cdot \underline{\pi}^{\infty}, \gamma\right)$ is an actual event.

Finally, $\mathbb{P}\left(\underline{\alpha} \cdot \underline{\pi}^{\infty}, \gamma\right)=\mathbb{R} \mathbb{P}\left(\underline{\alpha} \cdot[0]^{\infty}, \gamma_{1}\right)+\mathbb{P}\left(\underline{0}^{\ell}(\underline{\alpha}) \cdot \underline{\pi}^{\infty}, \gamma_{2}\right)-\mathbb{P}(\perp, \lambda n . n)$ by Propositions 2.12 and 2.7 and, by Propositions 2.16, 2.18, 2.15 and 2.7, this is equal to $\frac{\sum_{k=1}^{\ell(\pi)} \pi_{k}}{\ell(\underline{\pi})}$.

We conclude this section with the following result:

Theorem 2.20. Regular events form a boolean algebra with the operations inherited by the algebra of potential events.

Proof. First of all $\perp=[0]^{\infty}$ and $\top=[1]^{\infty}$. Suppose now that $\alpha \cdot \underline{\pi}^{\infty}$ and $\beta . \psi^{\infty}$ are regular events. Then $\neg\left(\underline{\alpha} . \underline{\pi}^{\infty}\right):=(\neg \underline{\alpha}) .(\neg \underline{\pi})^{\infty}$ where $\neg \underline{\alpha}$ and $\neg \underline{\pi}$ are obtained by changing each term $x$ of the finite lists to $1-x$. Without loss of generality, we can suppose that $\ell(\underline{\beta}) \geq \ell(\underline{\alpha})$. We have that:

$$
\begin{aligned}
& \underline{\alpha} \cdot \underline{\pi}^{\infty} \wedge \underline{\beta} \cdot \underline{\psi}^{\infty}=\underline{\gamma} \cdot \underline{\rho}^{\infty} \\
& \underline{\alpha} \cdot \underline{\pi}^{\infty} \vee \underline{\beta} \cdot \underline{\psi}^{\infty}=\underline{\gamma}^{\prime} \cdot \underline{\rho}^{\infty}
\end{aligned}
$$

where $\ell(\underline{\gamma})=\ell\left(\underline{\gamma^{\prime}}\right)=\ell(\underline{\beta}), \ell(\underline{\rho})=\ell\left(\underline{\rho^{\prime}}\right)=\ell(\underline{\pi}) \ell(\underline{\psi})$, and

$$
\begin{gathered}
\left\{\begin{array}{l}
\gamma_{i}:=\alpha_{i} \wedge \beta_{i} \text { if } i \leq \ell(\underline{\alpha}) \\
\gamma_{i}:=\pi_{\bmod (i-\ell(\underline{\alpha})-1, \ell(\underline{\pi}))+1} \wedge \beta_{i} \text { if } \ell(\underline{\alpha})<i \leq \ell(\underline{\beta})
\end{array}\right. \\
\left\{\begin{array}{l}
\gamma_{i}^{\prime}:=\alpha_{i} \vee \beta_{i} \text { if } i \leq \ell(\underline{\alpha}) \\
\gamma_{i}^{\prime}:=\pi_{\bmod (i-\ell(\underline{\alpha}-1, \ell(\underline{\pi}))+1} \vee \beta_{i} \text { if } \ell(\underline{\alpha})<i \leq \ell(\underline{\beta})
\end{array}\right. \\
\left\{\begin{array}{l}
\rho_{i}:=\pi_{\bmod (\ell(\underline{\beta})-\ell(\underline{\alpha})+i-1, \ell(\underline{\pi}))+1} \wedge \psi_{\bmod (i-1, \ell(\underline{\psi}))+1} \\
\rho_{i}^{\prime}:=\pi_{\bmod (\ell(\underline{\beta})-\ell(\underline{\alpha})+i-1, \ell(\underline{\pi}))+1} \vee \psi_{\bmod (i-1, \ell(\underline{\psi}))+1} \\
\text { for every } 1 \leq i \leq \ell(\underline{\pi}) \ell(\underline{\psi}) .
\end{array}\right.
\end{gathered}
$$

\section{Probabilistic versions of the limited principle of omniscience?}

The limited principle of omniscience, for short LPO, is a non-constructive principle which is weaker than the law of excluded middle (see e.g. [2]) which 
plays an important role in constructive reverse mathematics (see [4]). It can be formulated in our framework as follows

$$
\text { LPO : }(\forall \mathrm{e} \in \mathcal{P})\left(\left(\forall n \in \mathbb{N}^{+}\right)(\mathrm{e}(n)=0) \vee\left(\exists n \in \mathbb{N}^{+}\right)(\mathrm{e}(n)=1)\right)
$$

Having introduced a notion of probability $\mathbb{P}$ on (some) binary sequences, we can consider some variants of this principle by restricting it to some subsets of potential events or by modifying the disjunction in it.

First, we need some abbreviations:

1. List $^{+}(A)$ is the set of finite lists of elements from a set $A$ with positive length;

2. $\operatorname{Incr}\left(\mathbb{N}^{+}, \mathbb{N}^{+}\right)$is the set of strictly increasing sequences of positive natural numbers;

3. $\mathcal{A}:=\left\{\mathrm{e} \in \mathcal{P} \mid\left(\exists \gamma \in \operatorname{Incr}\left(\mathbb{N}^{+}, \mathbb{N}^{+}\right)\right)((\mathrm{e}, \gamma) \in \widetilde{\mathcal{A}})\right\}$

4. $\varphi(\mathbb{P}[\mathrm{e}])$ means e $\in \mathcal{A} \wedge\left(\forall \gamma \in \operatorname{Incr}\left(\mathbb{N}^{+}, \mathbb{N}^{+}\right)\right)((\mathrm{e}, \gamma) \in \widetilde{\mathcal{A}} \rightarrow \varphi(\mathbb{P}(\mathrm{e}, \gamma)))$ whenever $\varphi(x)$ is a proposition depending on a real number $x$;

5. $\mathcal{R}:=\left\{\mathrm{e} \in \mathcal{P} \mid(\exists \underline{\alpha} \in \operatorname{List}(\{0,1\}))\left(\exists \underline{\pi} \in \operatorname{List}^{+}(\{0,1\})\right)\left(\mathrm{e}=\mathcal{P} \underline{\alpha} . \underline{\pi}^{\infty}\right)\right\} ;$

6. $\mathcal{N}:=\left\{\mathrm{e} \in \mathcal{P} \mid \mathbb{P}[\mathrm{e}]=\mathbb{R}_{\mathbb{R}} 0\right\}$.

That is, $\mathcal{A}, \mathcal{R}$ and $\mathcal{N}$ are the subsets of $\mathcal{P}$ of actual, regular and null events, respectively.

Before proceeding, let us prove a simple, but very important fact.

Lemma 3.1. Let e $\in \mathcal{P}$;

1. if $\mathbb{P}[\mathrm{e}]>_{\mathbb{R}} 0$, then $\left(\exists n \in \mathbb{N}^{+}\right)(\mathrm{e}(n)=1)$;

2. if $\left(\forall n \in \mathbb{N}^{+}\right)(\mathrm{e}(n)=0)$, then $\mathbb{P}[\mathrm{e}]=\mathbb{R} 0$.

Proof. 2. holds since $\left(\forall n \in \mathbb{N}^{+}\right)(\mathrm{e}(n)=0)$ means exactly $\mathrm{e}=\mathcal{P} \perp$.

Suppose now that $(e, \gamma) \in \widetilde{\mathcal{A}}$ and $\mathbb{P}(e, \gamma)>_{\mathbb{R}} 0$. As a consequence of the definition of $>_{\mathbb{R}}$, there exists $m \in \mathbb{N}^{+}$such that $\mathbb{P}(\mathrm{e}, \gamma)(m)>\frac{1}{m}$, i.e.

$$
\sum_{i=1}^{\gamma(m)} m \mathrm{e}(i)>\gamma(m)>0 .
$$

Hence there exists $n$ such that $1 \leq n \leq \gamma(m)$ and $\mathrm{e}(n)=1$.

Thus $\left(\exists n \in \mathbb{N}^{+}\right)(\mathrm{e}(n)=1)$ and 1 . is proved. 
We can now introduce a family of "probabilistic" versions of LPO.

Definition 3.2. For every subset $\mathcal{E}$ of $\mathcal{P}$ we define the following principles:

1. $\mathbf{L P O}[\mathcal{E}] \equiv{ }^{\operatorname{def}}(\forall \mathrm{e} \in \mathcal{E})\left[\left(\forall n \in \mathbb{N}^{+}\right)(\mathrm{e}(n)=0) \vee\left(\exists n \in \mathbb{N}^{+}\right)(\mathrm{e}(n)=1)\right]$.

2. $\mathbb{P}-\mathbf{L P O}[\mathcal{E}] \equiv{ }^{\operatorname{def}}(\forall \mathrm{e} \in \mathcal{E})\left[\mathbb{P}[\mathrm{e}]=_{\mathbb{R}} 0 \vee\left(\exists n \in \mathbb{N}^{+}\right)(\mathrm{e}(n)=1)\right]$.

3. $\mathbb{P P}-\mathbf{L P O}[\mathcal{E}] \equiv{ }^{\operatorname{def}}(\forall \mathrm{e} \in \mathcal{E})\left[\mathbb{P}[\mathrm{e}]=_{\mathbb{R}} 0 \vee \mathbb{P}[\mathrm{e}]>_{\mathbb{R}} 0\right]$.

Notice that $\mathbf{L P O}[\mathcal{P}]$ is equivalent to $\mathbf{L P O}$, and that $\mathbb{P P}-\mathbf{L P O}[\mathcal{P}]$ implies $\mathcal{P}=\mathcal{A}$.

Let us now study the relation between these probabilistic versions of LPO when $\mathcal{E}$ is $\mathcal{P}, \mathcal{A}, \mathcal{N}$ or $\mathcal{R}$. As a direct consequence of Lemma 3.1 we have:

Lemma 3.3. If $\mathcal{E}$ is a subset of $\mathcal{P}$, then

1. $\mathbb{P P}-\mathbf{L P O}[\mathcal{E}] \rightarrow \mathbb{P}-\mathbf{L P O}[\mathcal{E}]$.

2. $\mathbf{L P O}[\mathcal{E}] \rightarrow \mathbb{P}-\mathbf{L P O}[\mathcal{E}]$.

Moreover, as a consequence of the definitions we have that

Lemma 3.4. If $\mathcal{E} \subseteq \mathcal{E}^{\prime}$ are subsets of $\mathcal{P}$, then

1. $\mathbf{L P O}\left[\mathcal{E}^{\prime}\right] \rightarrow \operatorname{LPO}[\mathcal{E}]$.

2. $\mathbb{P}-\mathbf{L P O}\left[\mathcal{E}^{\prime}\right] \rightarrow \mathbb{P}-\mathbf{L P O}[\mathcal{E}]$.

3. $\mathbb{P P}-\mathbf{L P O}\left[\mathcal{E}^{\prime}\right] \rightarrow \mathbb{P P}-\mathbf{L P O}[\mathcal{E}]$.

Some of these principles can be proven constructively, while one is constructively false:

Lemma 3.5. The following hold:

1. $\operatorname{LPO}[\mathcal{R}]$.

2. $\mathbb{P}-\mathbf{L P O}[\mathcal{R}]$.

3. $\mathbb{P P}-\mathbf{L P O}[\mathcal{R}]$.

4. $\mathbb{P P}-\mathbf{L P O}[\mathcal{N}]$.

5. $\mathbb{P}-\mathbf{L P O}[\mathcal{N}]$. 
6. $\neg \mathbb{P P}-\mathbf{L P O}[\mathcal{P}]$.

Proof. Let us prove one by one of the statements above.

1. $\mathbf{L P O}[\mathcal{R}]$ holds: if $\mathrm{e}=\mathcal{P} \underline{\alpha} \cdot \underline{\pi}^{\infty}$ is in $\mathcal{R}$, then

$$
\left(\forall n \in \mathbb{N}^{+}\right)(\mathrm{e}(n)=0) \vee\left(\exists n \in \mathbb{N}^{+}\right)(\mathrm{e}(n)=1)
$$

is equivalent to $\sum_{i=1}^{\ell(\underline{\alpha})} \alpha_{i}+\sum_{j=1}^{\ell(\underline{\pi})} \pi_{j}=0 \vee \sum_{i=1}^{\ell(\underline{\alpha})} \alpha_{i}+\sum_{j=1}^{\ell(\underline{\pi})} \pi_{j}>0$.

Since equality of natural numbers is decidable, we can conclude.

2. This is a consequence of 1 . and Lemma 3.3.

3. If $\mathrm{e}=\mathcal{P} \underline{\alpha} \cdot \underline{\pi}^{\infty}$ is in $\mathcal{R}$, then $\mathbb{P}[\mathrm{e}]=\mathbb{R} \frac{\sum_{j=1}^{\ell(\underline{\pi})} \pi_{j}}{\ell(\underline{\pi})} \in \mathbb{Q}$ and we can thus decide whether $\mathbb{P}[\mathrm{e}]=\mathbb{R} 0$ or $\mathbb{P}[\mathrm{e}]>_{\mathbb{R}} 0$.

4. This is obvious, since $\mathrm{e} \in \mathcal{N}$ means, by definition, that $\mathbb{P}[\mathrm{e}]=\mathbb{R} 0$.

5. This is a consequence of 4. and Lemma 3.3.

6. There exist potential events e for which there is no $\gamma$ such that $(\mathrm{e}, \gamma) \in$ $\widetilde{\mathcal{A}}$. Consider for example the sequence e which starts with a 1 and a 0 and then alternates a group of $2 \cdot 3^{n}$ ones and a group of $2 \cdot 3^{n}$ zeros increasing $n$ at each step

$101100111111000000 \ldots$

In this case $\Phi\left(2 \cdot 3^{n}\right)=\frac{1}{2}$ and $\Phi\left(4 \cdot 3^{n}\right)=\frac{3}{4}$ for every $n \in \mathbb{N}^{+}$.

After these first three lemmas, the situation about the remaining variants can be summarize as follows

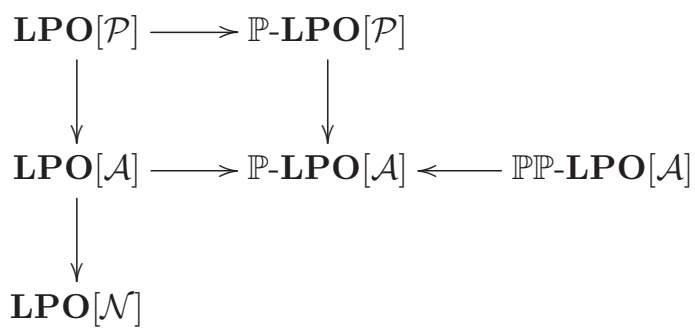

However, the left side of the previous diagram collapses: 
Lemma 3.6. $\mathbf{L P O}[\mathcal{N}]$ implies $\mathbf{L P O}[\mathcal{P}]$. Hence $\mathbf{L P O}[\mathcal{P}], \mathbf{L P O}[\mathcal{A}]$ and $\mathbf{L P O}[\mathcal{N}]$ are all equivalent.

Proof. Let e be a potential event. We can define a non-decreasing sequence $\widetilde{e}$ in $\mathcal{P}$ as follows:

$$
\left\{\begin{array}{l}
\widetilde{\mathrm{e}}(1):=\mathrm{e}(1) \\
\widetilde{\mathrm{e}}(n+1):=\widetilde{\mathrm{e}}(n) \vee \mathrm{e}(n+1)\left[n \in \mathbb{N}^{+}\right] .
\end{array}\right.
$$

As a consequence of the definition of $\widetilde{\mathrm{e}}$,

1. $\left(\exists n \in \mathbb{N}^{+}\right)(\mathrm{e}(n)=1)$ if and only if $\left(\exists n \in \mathbb{N}^{+}\right)(\widetilde{\mathrm{e}}(n)=1)$, and

2. $\left(\forall n \in \mathbb{N}^{+}\right)(\mathrm{e}(n)=0)$ if and only if $\left(\forall n \in \mathbb{N}^{+}\right)(\widetilde{\mathrm{e}}(n)=0)$.

Using $\widetilde{\mathrm{e}}$ we define another potential event $\alpha(\widetilde{\mathrm{e}})$ as follows:

$$
\left\{\begin{array}{l}
\alpha(\widetilde{\mathrm{e}})(1):=\widetilde{\mathrm{e}}(1) \\
\alpha(\widetilde{\mathrm{e}})(n+1):=\widetilde{\mathrm{e}}(n+1)-\widetilde{\mathrm{e}}(n)\left[n \in \mathbb{N}^{+}\right]
\end{array}\right.
$$

Since $\widetilde{e}$ is non-decreasing, $\alpha(\widetilde{\mathrm{e}})$ takes value 1 for at most one input. In particular $\alpha(\widetilde{\mathrm{e}})$ is in $\mathcal{N}$,

$$
\left(\exists n \in \mathbb{N}^{+}\right)(\widetilde{\mathrm{e}}(n)=1) \text { if and only if }\left(\exists n \in \mathbb{N}^{+}\right)(\alpha(\widetilde{\mathrm{e}})(n)=1)
$$

and

$$
\left(\forall n \in \mathbb{N}^{+}\right)(\widetilde{\mathrm{e}}(n)=0) \text { if and only if }\left(\forall n \in \mathbb{N}^{+}\right)(\alpha(\widetilde{\mathrm{e}})(n)=0) .
$$

If $\mathbf{L P O}[\mathcal{N}]$ holds, then

$$
\left(\exists n \in \mathbb{N}^{+}\right)(\alpha(\widetilde{\mathrm{e}})(n)=1) \vee\left(\forall n \in \mathbb{N}^{+}\right)(\alpha(\widetilde{\mathrm{e}})(n)=0),
$$

which is equivalent to $\left(\exists n \in \mathbb{N}^{+}\right)(\mathrm{e}(n)=1) \vee\left(\forall n \in \mathbb{N}^{+}\right)(\mathrm{e}(n)=0)$, holds. Thus from $\mathbf{L P O}[\mathcal{N}]$ follows $\mathbf{L P O}[\mathcal{P}]$.

After the proof of this lemma the situation is the following:

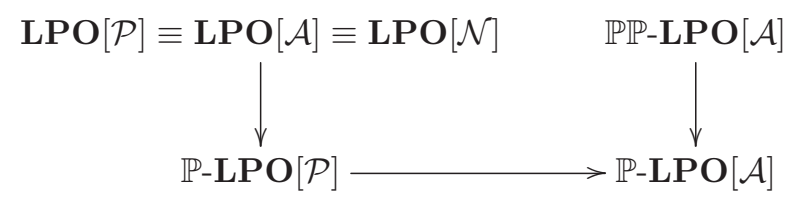

Lemma 3.7. $\mathbb{P}-\mathbf{L P O}[\mathcal{A}]$ implies LPO $[\mathcal{P}]$, thus $\mathbb{P}-\mathbf{L P O}[\mathcal{A}], \mathbb{P}-\mathbf{L P O}[\mathcal{P}]$ and $\mathbf{L P O}[\mathcal{P}]$ are equivalent. 
Proof. Let e be a potential event and let $\varphi: \mathbb{N}^{+} \rightarrow \mathbb{N}$ be the surjective primitive recursive function sending each positive natural number $n$ to the exponent of 2 in its prime factorization.

Let $\widehat{e}$ be the potential event defined by

$$
\widehat{\mathrm{e}}(n):=\mathrm{e}(\varphi(n)+1)\left[n \in \mathbb{N}^{+}\right] .
$$

We have that $\left(\exists n \in \mathbb{N}^{+}\right)(\widehat{\mathrm{e}}(n)=1)$ if and only if $\left(\exists n \in \mathbb{N}^{+}\right)(\mathrm{e}(n)=1)$. Moreover $\widehat{\mathrm{e}} \in \mathcal{A}$, because $\left(\widehat{\mathrm{e}}, \lambda n .2^{n+2}\right) \in \widetilde{\mathcal{A}}$. Since for every $n>3$

$$
\left(\mathbb{P}\left(\widehat{\mathrm{e}}, \lambda n .2^{n+2}\right)\right)(n-3)=\frac{\mathrm{e}(n)}{2^{n-1}}+\sum_{k=1}^{n-1} \frac{\mathrm{e}(k)}{2^{k}}
$$

it follows that $\mathbb{P}[\hat{\mathrm{e}}]=\mathbb{R}_{\mathbb{R}} \sum_{n=1}^{+\infty} \frac{\mathrm{e}(n)}{2^{n}}$.

In particular, $\mathbb{P}[\hat{\mathrm{e}}]=_{\mathbb{R}} 0$ if and only if $\left(\forall n \in \mathbb{N}^{+}\right)(\mathrm{e}(n)=0)$.

Assuming $\mathbb{P}$-LPO $[\mathcal{A}]$, we obtain $\mathbb{P}(\widehat{\mathrm{e}})=0 \vee\left(\exists n \in \mathbb{N}^{+}\right)(\widehat{\mathrm{e}}(n)=1)$ which is equivalent to $\left(\exists n \in \mathbb{N}^{+}\right)(\mathrm{e}(n)=1) \vee\left(\forall n \in \mathbb{N}^{+}\right)(\mathrm{e}(n)=0)$.

We have hence shown that $\mathbb{P}-\mathbf{L P O}[\mathcal{A}]$ implies $\mathbf{L P O}[\mathcal{P}]$.

After the proof of this lemma the situation is the following:

$\mathbb{P P}-\mathbf{L P O}[\mathcal{A}] \rightarrow \mathbf{L P O}[\mathcal{P}](\equiv \mathbf{L P O}[\mathcal{A}] \equiv \mathbf{L P O}[\mathcal{N}] \equiv \mathbb{P}-\mathbf{L P O}[\mathcal{P}] \equiv \mathbb{P}-\mathbf{L P O}[\mathcal{A}])$

However we have the following

Lemma 3.8. LPO $[\mathcal{P}]$ implies $\mathbb{P P}$-LPO $[\mathcal{A}]$.

Proof. Assume $\mathbf{L P O}[\mathcal{P}]$. Since we have assumed countable choice, trichotomy for Bishop reals follows (see [2]) and thus $\mathbb{P P}-\mathbf{L P O}[\mathcal{A}]$ holds.

As consequence of all the lemmas above we have the following

Theorem 3.9.

$$
\mathbf{L P O}[\mathcal{P}] \equiv \mathbf{L P O}[\mathcal{A}] \equiv \mathbf{L P O}[\mathcal{N}] \equiv \mathbb{P}-\mathbf{L P O}[\mathcal{P}] \equiv \mathbb{P}-\mathbf{L P O}[\mathcal{A}] \equiv \mathbb{P P}-\mathbf{L P O}[\mathcal{A}]
$$

Thus we have proved that no one of the variations on LPO which we have introduced is different from a true statement, a false statement or LPO itself. Thus, from one side the hope of having an authentic "probabilistic" variant of $\mathbf{L P O}$ has failed; however the results above provide new statements equivalent to $\mathbf{L P O}$, namely $\mathbf{L P O}[\mathcal{A}], \mathbf{L P O}[\mathcal{N}], \mathbb{P}-\mathbf{L P O}[\mathcal{A}], \mathbb{P}-\mathbf{L P O}[\mathcal{P}]$ and $\mathbb{P P}-\mathbf{L P O}[\mathcal{A}]$.

\section{Acknowledgements}

The author would like to thank Francesco Ciraulo, Hannes Diener, Milly Maietti, Fabio Pasquali and Giovanni Sambin for fruitful discussions about the subject of this paper. The author thanks EU-MSCA-RISE project 731143 "Computing with Infinite Data" (CID) for supporting the research. 


\section{References}

[1] E. Bishop, D. S. Bridges, Constructive analysis, Springer 1985.

[2] D. Bridges, F. Richman, Varieties of Constructive Mathematics, Cambridge University Press 1987.

[3] Y.K. Chan, Notes on constructive probability theory, Ann. Probability 2 (1974), 51-75

[4] H. Ishihara, Reverse Mathematics in Bishop's Constructive Mathematics, Philosophia Scientae (2006), 43-59.

[5] M.E. Maietti, A minimalist two-level foundation for constructive mathematics, $A n$ nals of Pure and Applied Logic 160:3 (2009), 319-354.

[6] M.E. Maietti, G. Sambin, Toward a minimalist foundation for constructive mathematics, In: From Sets and Types to Topology and Analysis: Practicable Foundations for Constructive Mathematics (Eds. L. Crosilla, P. Schuster), Oxford University Press 2005, pp. 91-114.

[7] I. Niven, The asymptotic density of sequences, Bull. Amer. Math. Soc. 6:11 (1951), 420-434.

Dipartimento di Matematica "Tullio Levi-Civita"

Università di Padova

35131 Padova (PD), Italy

maschio@math.unipd.it 